\title{
Simulation model of self-organizing pedestrian movement considering following behavior*
}

\author{
Zhilu YUAN ${ }^{1}$, Hongfei JIA ${ }^{\dagger 1}$, Mingjun LIAO ${ }^{2}$, Linfeng ZHANG ${ }^{1}$, \\ Yixiong FENG ${ }^{3}$, Guangdong TIAN ${ }^{\dagger \dagger 1}$ \\ ( ${ }^{1}$ School of Transportation, Jilin University, Changchun 130012, China) \\ ( ${ }^{2}$ Department of Civil Engineering, Beihua University, Jilin 132013, China) \\ $\left({ }^{3}\right.$ The State Key Lab of Fluid Power Transmission and Control, Zhejiang University, Hangzhou 310027, China) \\ †E-mail: jiahf@jlu.edu.cn; tiangd2013@163.com \\ Received Sept. 28, 2016; Revision accepted Nov. 22, 2016; Crosschecked Aug. 19, 2017
}

\begin{abstract}
A new force is introduced in the social force model (SFM) for computing following behavior in pedestrian counterflow, whereby an individual tries to approach others in the same direction to avoid conflicts with pedestrians from the opposite direction. The force, like a kind of gravitation, is modeled based on the movement state and visual field of the pedestrian, and is added to the classical SFM. The modified model is presented to study the impact of following behavior on the process of lane formation, the conflict, the number of lanes formed, and the traffic efficiency in the simulations. Simulation results show that the following behavior has a significant effect on the phenomenon of lane formation and the traffic efficiency.
\end{abstract}

Key words: Gravitation; Pedestrian counterflow; Social force model (SFM); Lane formation; Self-organizing http://dx.doi.org/10.1631/FITEE.1601592

CLC number: TP391.9; U698.2

\section{Introduction}

Previous studies showed that the pedestrian flow has self-organization characteristics from the macroscopic point of view (Lam et al., 2002; Seyfried et al., 2005; Fujiyama and Tyler, 2009; Iryo-Asano et al., 2015; Li et al., 2015; Jia et al., 2016; Tang et al., 2017). Typical self-organized phenomena of pedestrian flow include arch-like formation, bottleneck oscillation, lane formation, and stripe formation. The results of research on the self-organizing characteristics of pedestrian flow have great significance for evacuation analysis, passenger transport hub design, and traffic control.

\section{$\ddagger$ Corresponding author}

* Project supported by the National Natural Science Foundation of China (Nos. 51278221 and 51378076), the Chinese Postdoctoral Science Foundation (Nos. 2015M571369 and 2012M511343), and Jilin Science and Technology Development Program, China (Nos. 20140204027SF and 20170101155JC)

(ib) ORCID: Zhi-lu YUAN, http://orcid.org/0000-0002-7431-6599 (C)Zhejiang University and Springer-Verlag GmbH Germany 2017
Many researchers use discrete models, such as the cellular automaton model (CA) (Zhang et al., 2004; Liao and Liu, 2015) and the lattice gas model (Chen et al., 2009), to study the self-organizing phenomena of pedestrian flow. In CA, the factors influencing lane formation are: pedestrian walking habits under subconscious control (Yue et al., 2010; Li et al., 2017), pedestrian visual effect (Tajima et al., 2002), and the effect of prediction (Wang et al., 2012). The $k$-nearest-neighbors around pedestrians were considered from the point of the psychophysiological and surrounding environment (Ma et al., 2010).

Some researchers applied the continuous model to study the pedestrian self-organizing phenomenon. Helbing $(1996$; 2001) and Helbing et al. (2000) studied the oscillation and arch-like formation in the early social force model (SFM). The following behavior (a important factor of lane formation) under the panic condition was also studied by introducing a panic 
factor. They pointed out that the lane formation can be simulated even without assuming a preference for any side. They considered that the essential cause of lane formation should be the higher relative velocity of pedestrians, which contributes to the severity of physical contact in the opposite direction (Helbing et al., 2002). SFM can produce trail formation and create lines of pedestrians with the same direction (Helbing and Molnár, 1995; Werner and Helbing, 2003). Therefore, SFM is suitable for studying the lane formation phenomenon.

However, some researchers pointed out that there are also some drawbacks for SFM for studying the lane formation phenomenon. Some researchers improved SFM by developing separate force models for collision avoidance between pedestrians. Collision avoidance behaviors were adopted to SFM by adding either a component to the desired velocity (Smith et al., 2009) or a new force to change the pedestrian trajectories (Pelechano et al., 2007). Heliövaara et al. (2012) presented a new combined model based on SFM, which alters the desired moving direction of the pedestrian and takes into account both collision avoidance and lane formation. Guo (2014) considered the influence of position factors (between the pedestrian and the entrance/exit boundary) on the counter flow and the lane formation. These studies analyzed the lane formation phenomenon of pedestrian flow from different perspectives. The modified models are all based on the fundamental SFM and consider just the different pedestrian behavior characteristics in the process of avoiding collisions.

In recent years, many researchers have concentrated on following behavior. For example, the pedestrian waiting probability was introduced into the lattice gas mode model to study the following behavior (Li et al., 2005; Weng et al., 2006; Yang et al., 2008). Helbing et al. (2005) analyzed following behavior in the panic and unfamiliar environment, which is a kind of herding behavior. Kuang et al. (2010) analyzed the effect of different levels of following behavior strength on the opposite pedestrian flow. Based on video observation, it is found that the following behavior is very common in a normal condition (see Fig. 1 for an example).

Following behavior in the counterflow means that an individual tries to approach the others in the same direction to avoid conflicts with the opposing pedestrians. Following behavior reflects pedestrian self-organizing characteristics irrelevant to the level of socialization (Saloma et al., 2003). In the counterflow, with density increase, pedestrians would initiatively adjust their own walking strategy to avoid possible collisions. When pedestrian's free movement is limited, group consciousness would affect the pedestrian walking strategy, and then pedestrians tend to follow the pedestrians ahead in the same direction. The kind of following behavior of the pedestrian is one of the important factors in the pedestrian selforganizing phenomenon.

The primary objective of this research is to study the proposed gravitation model of following behavior, which is affected by motion state (position and velocity) of pedestrians ahead, and its effects on lane formation. This paper is divided into two sections, making up a simulation on the pedestrian lane formation. In the first section we analyze the original SFM, and build a gravitation model that reflects pedestrian following behavior. In the second section we present several cases studying the following behavior of pedestrians.

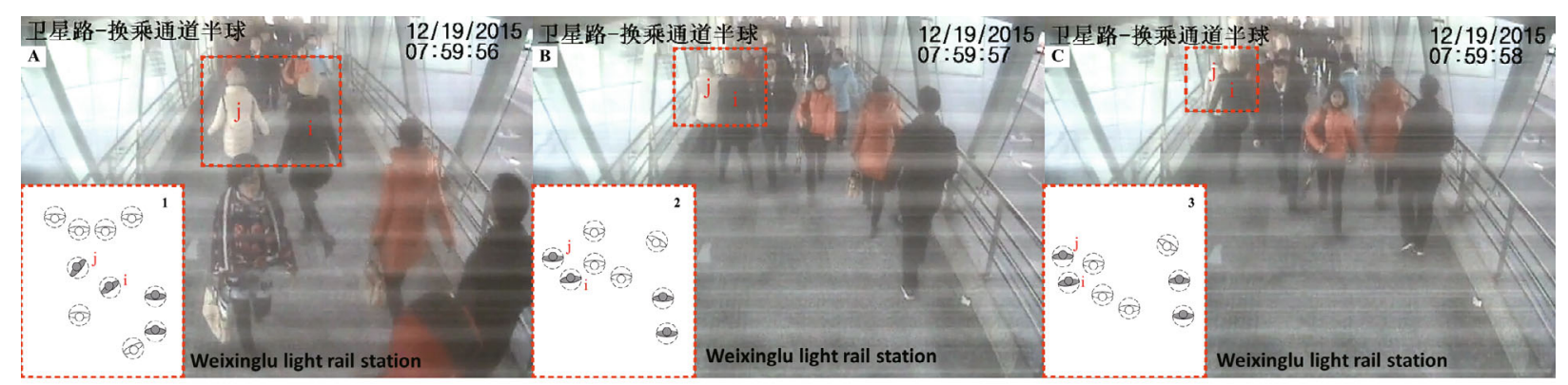

Fig. 1 Following behaviors shown in video 


\section{Model}

\subsection{Analysis of the social force model}

Denote $\boldsymbol{v}_{i}, \boldsymbol{f}_{\text {will }}, \boldsymbol{f}_{i, \text { ped }}$, and $\boldsymbol{f}_{i, \text { wal }}$ as the velocity of pedestrian $i$, his/her will force toward the target, interaction force between pedestrian $i$ and other pedestrians, and interaction force between pedestrian $i$ and the walls and boundaries, respectively. The original SFM for pedestrian traffic flow is (Helbing et al., 2000) (Fig. 2)

$$
m_{i} \frac{\mathrm{d} \boldsymbol{v}_{i}}{\mathrm{~d} t}=\boldsymbol{f}_{\text {will }}+\boldsymbol{f}_{i, \text { ped }}+\boldsymbol{f}_{i, \text { wal }},
$$

where $m_{i}$ is the mass of pedestrian $i$. SFM considers that a pedestrian likes to move with a certain desired speed $v_{i}^{0}$ in a certain direction $\boldsymbol{e}_{i}^{0}$, and therefore tends to correspondingly adapt his/her actual velocity $\boldsymbol{v}_{i}$ within the so-called relaxation time $\tau_{i}$ :

$$
\boldsymbol{f}_{\mathrm{will}}=m_{i} \frac{v_{i}^{0}(t) \boldsymbol{e}_{i}^{0}(t)-\boldsymbol{v}_{i}(t)}{\tau_{i}}
$$

The pedestrian motion speed is affected by not only the will force, but also the interaction forces, such as the repulsive force between two pedestrians $i$ and $j\left(\boldsymbol{f}_{i j}\right)$ and the repulsive force between pedestrian $i$ and the wall or boundary $\left(\boldsymbol{f}_{i w}\right)$. Thus,

$$
\begin{aligned}
& \begin{array}{r}
\boldsymbol{f}_{i, \text { ped }}= \\
=\sum_{j(\neq i)} \boldsymbol{f}_{i j}
\end{array} \\
&=\sum_{j(\neq i)}\left\{\left[A_{i} \exp \left(\left(r_{i j}-d_{i j}\right) / B_{i}\right)+k g\left(r_{i j}-d_{i j}\right)\right] \boldsymbol{n}_{i j}\right. \\
&\left.\quad+\kappa g\left(r_{i j}-d_{i j}\right) \Delta v_{j i} \boldsymbol{t}_{i j}\right\}, \\
& \boldsymbol{f}_{i, \text { wal }}=\sum_{w} \boldsymbol{f}_{i w} \\
&=\sum_{w}\left\{\left[A_{i} \exp \left(\left(r_{i}-d_{i w}\right) / B_{i}\right)+k g\left(r_{i}-d_{i w}\right)\right] \boldsymbol{n}_{i w}\right. \\
&\left.\quad-\kappa g\left(r_{i}-d_{i w}\right)\left(\boldsymbol{v}_{i} \cdot \boldsymbol{t}_{i w}\right) \boldsymbol{t}_{i w}\right\},
\end{aligned}
$$

where $A_{i}$ and $B_{i}$ are positive constants, $r_{i j}$ is the sum of the radii of pedestrians $r_{i}$ and $r_{j}, d_{i j}=\left\|\boldsymbol{d}_{i}-\boldsymbol{d}_{j}\right\|$ denotes the distance between the centers of two pedestrians. $\boldsymbol{n}_{i j}$ is the normalized vector pointing from pedestrian $j$ to $i, \boldsymbol{t}_{i j}$ is a tangential direction, and $\Delta v_{j i}=\left(\boldsymbol{v}_{j}-\boldsymbol{v}_{i}\right) \cdot \boldsymbol{t}_{i j}$. The function of $g(x)$ equals zero if the pedestrians do not touch each other $\left(d_{i j}>r_{i j}\right)$, and equals the argument $x$ otherwise. $d_{i w}$ denotes the distance between wall $w$ and pedestrian $i, \boldsymbol{n}_{i w}$ is the normalized vector pointing

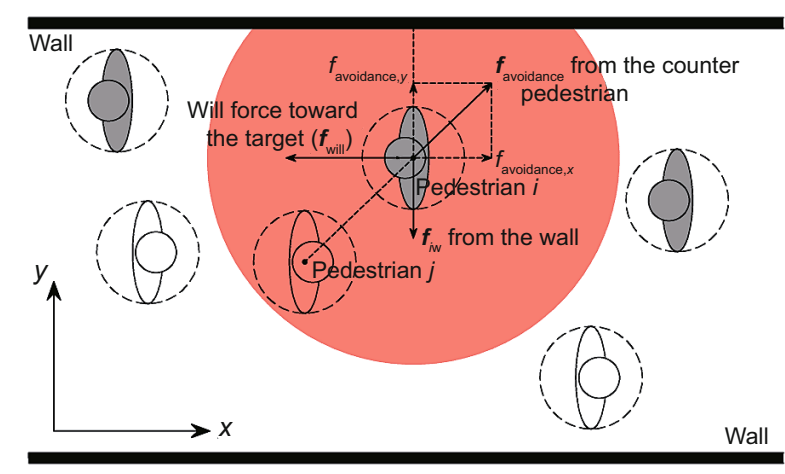

Fig. 2 Force analysis of the pedestrian collision avoidance process in the original social force model (References to color refer to the online version of this figure)

from wall $w$ to pedestrian $i$, and $\boldsymbol{t}_{i w}$ is a tangential direction.

We focus on the collision avoidance behavior of pedestrians and thus study mainly the interaction force between two pedestrians $\left(\boldsymbol{f}_{i j}\right) . \boldsymbol{f}_{i j}$ can be divided into three parts:

$$
\boldsymbol{f}_{\text {avoidance }}=A_{i} \exp \left(\left(r_{i j}-d_{i j}\right) / B_{i}\right) \boldsymbol{n}_{i j},
$$

and two contact forces

$$
\begin{aligned}
\boldsymbol{f}_{\text {extrusion }} & =k g\left(r_{i j}-d_{i j}\right) \boldsymbol{n}_{i j}, \\
\boldsymbol{f}_{\text {friction }} & =\kappa g\left(r_{i j}-d_{i j}\right) \Delta v_{j i} \boldsymbol{t}_{i j} .
\end{aligned}
$$

$\boldsymbol{f}_{\text {avoidance }}$ describes the psychological tendency of two pedestrians $i$ and $j$ to keep a velocity-dependent distance from each other. When $d_{i j} \leq r_{i j}$, there exists a physical contact between pedestrians, i.e., $\boldsymbol{f}_{\text {extrusion }}$ and $\boldsymbol{f}_{\text {friction. }}$.

Applying SFM to simulate the lane formation phenomenon, it is possible to capture the phenomenon from the macro perspective and reflect the awareness of pedestrians in protecting their own walking space. SFM can simulate the lane formation phenomenon, but it cannot simulate other behaviors, such as following behavior. As shown in Fig. 2, pedestrian $i$ is moving from the right end to the left in the passage. The red area is the $\boldsymbol{f}_{\text {avoidance }}$ search range of pedestrian $i$; pedestrian $j$ is within the search range and his/her movement direction is opposite to that of pedestrian $i$. Collision avoidance between pedestrians $i$ and $j$ relies on $\boldsymbol{f}_{\text {avoidance }}$ between $i$ and $j$. $\boldsymbol{f}_{\text {avoidance }}$ can be divided into a horizontal component $f_{\text {avoidance, } x}$ and a vertical component $f_{\text {avoidance, } y}$. Under the action of $f_{\text {avoidance, } x}$, pedestrian $i$ begins to slow down. $f_{\text {avoidance, } y}$ determines the direction of collision avoidance. The 
direction of $f_{\text {avoidance, } y}$ is determined by the relative position between pedestrians, and the value of $f_{\text {avoidance, } y}$ will affect the duration of the collision avoidance process.

We find that in SFM the direction of collision avoidance is determined by the relative position between pedestrians and the velocity of pedestrians. SFM does not consider the location or the motion states of other pedestrians in the same direction, so it is unable to reflect the effect of following behavior on the direction of collision avoidance.

\subsection{Gravitation model}

Considering the pedestrian overlapping problem, Lakoba et al. (2005) improved the original SFM by setting reasonable parameters in the model. They set a rigid space for one pedestrian with the maximum space compression ratio as $S_{\mathrm{MAX}}=20 \%$. In this study, the concept of the rigid space and the parameters are introduced, and a visual field factor is introduced to increase the search scope of the original model.

To simulate the following behavior, we propose a new force $\boldsymbol{f}_{i \text {, gra }}$ named 'gravitation'. Some studies (Löhner, 2010) pointed out that in SFM, the order of the magnitudes of the forces is

$$
f_{\text {will }}<f_{\text {avoidance }}<f_{\text {ped }}<f_{\text {wal }} \text {. }
$$

We suppose that the gravitation and the will force have the same order. Thus,

$$
\boldsymbol{f}_{i, \text { gra }}=f_{i, \max } \sum_{j \neq i} \beta_{i j, 1} \beta_{i j, 2} \beta_{i j, 3} \beta_{i j, 4} \beta_{i j, 5} \beta_{i j, 6} \boldsymbol{n}_{i j},
$$

where $f_{i, \max }$ is the order of gravitation, and $\beta_{i j, n}$ $(n=1,2, \ldots, 6)$ are six non-dimensional parameters. $f_{i, \max }$ and $\beta_{i j, n}$ will be discussed in the following.

The order of gravitation is the same as that of the will force. Thus, we define

$$
f_{i, \max }=\phi m_{i} \frac{v_{i}^{0}}{\tau_{i}}
$$

where $\phi$ is the revise parameter which will be discussed in Section 3.

First, we define the concept of the search vision scope in the gravitation model. The scope of pedestrian vision is determined by two parts: vision radius $\left(l_{i}\right)$ and vision angle. If the distance between pedestrians $i$ and $j\left(d_{i j}\right)$ is larger than the vision radius of pedestrian $i$, or pedestrian $j$ is not in the vision angle of pedestrian $i$, the gravitation of pedestrian $j$ towards pedestrian $i$ will not exist. Normally, a pedestrian is concerned only with the pedestrians and barrier in front of him/her, so we define the vision angle as $\pi$ in the current direction of pedestrian $i$. If the angle between the current velocity of pedestrian $i\left(\boldsymbol{v}_{i}\right)$ and the vector from $i$ to $j\left(\boldsymbol{n}_{j i}\right)$ is less than or equal to $\pi / 2$, and $d_{i j}$ is less than or equal to $l_{i}$, pedestrian $j$ is within the vision of pedestrian $i$. Based on the above analysis, $\beta_{i j, 1}$ and $\beta_{i j, 2}$ are defined as

$$
\begin{gathered}
\beta_{i j, 1}= \begin{cases}1, & d_{i j} \leq l_{i}, \\
0, & d_{i j}>l_{i},\end{cases} \\
\beta_{i j, 2}= \begin{cases}1, & \frac{\boldsymbol{v}_{i} \cdot \boldsymbol{n}_{j i} d_{i j}}{v_{i} d_{i j}}>\cos \frac{\pi}{2}, \\
0, & \text { otherwise. }\end{cases}
\end{gathered}
$$

We assume that pedestrians are more inclined to follow the people whose walking direction is closer to his/her own expected direction. The higher the proximity between the current speed direction of pedestrian $j$ and the moving target direction $\left(\boldsymbol{e}_{i}^{0}\right)$ of pedestrian $i$ is, the more pedestrian $i$ is willing to follow pedestrian $j$, and the larger the gravitation on pedestrian $i$ is. If the angle $\left(\left\langle\boldsymbol{e}_{i}^{0}, \boldsymbol{v}_{j}\right\rangle\right)$ between the movement direction of pedestrian $j$ directly ahead of pedestrian $i$ and the target direction of pedestrian $i$ is larger than $\pi / 2$, pedestrian $i$ will not be attracted by pedestrian $j$. Based on the analysis above, $\beta_{i j, 3}$ is defined as

$$
\beta_{i j, 3}= \begin{cases}0, & \boldsymbol{e}_{i}^{0} \cdot \boldsymbol{v}_{j}<0 \\ \cos \left(\left\langle\boldsymbol{e}_{i}^{0}, \boldsymbol{v}_{j}\right\rangle\right)=\frac{\boldsymbol{e}_{i}^{0} \cdot \boldsymbol{v}_{j}}{e_{i}^{0} v_{j}}, & \boldsymbol{e}_{i}^{0} \cdot \boldsymbol{v}_{j} \geq 0\end{cases}
$$

Pedestrians are more likely to follow the people whose speed is higher. It is conducive for the pedestrian to quickly get rid of walking dilemma. The higher the speed of pedestrian $j\left(v_{j}\right)$, the larger the gravitation towards pedestrian $i$. The value of the gravitation between pedestrians has a certain limit; i.e., when $v_{j}$ is higher than the desired speed of pedestrian $i\left(v_{i}^{0}\right)$, the gravitation towards pedestrian $i$ will be small. Thus, $\beta_{i j, 4}$ is defined as

$$
\beta_{i j, 4}= \begin{cases}1, & v_{j}>v_{i}^{0}, \\ v_{j} / v_{i}^{0}, & v_{j} \leq v_{i}^{0} .\end{cases}
$$

The gravitation is also affected by the distance between pedestrians just like the avoidance force. 
The smaller the distance between pedestrians $j$ and $i$, the greater the impact of pedestrian $j$ on pedestrian $i$. Because the scope of pedestrian's vision is limited, in the limited scope, the smaller the distance between pedestrians $j$ and $i\left(d_{i j}-\left(r_{i}+r_{j}\right)\right)$, the more the attention that pedestrian $i$ will pay to pedestrian $j$. If the distance between pedestrians is larger than the vision range, the gravitation will not exist. Furthermore, when there is a physical contact between pedestrians $i$ and $j\left(d_{i j} \leq r_{i}+r_{j}\right)$, the gravitation will not continue to increase. So, we define $\beta_{i j, 5}$ as

$$
\beta_{i j, 5}= \begin{cases}1, & d_{i j} \leq r_{i}+r_{j} \\ \exp \left(-\frac{d_{i j}-\left(r_{i}+r_{j}\right)}{C_{i}}\right), & d_{i j}>r_{i}+r_{j} .\end{cases}
$$

The following behavior occurs when the pedestrians with free speed run into obstacles; namely, $v_{i}<v_{i}^{0}$. Based on the analysis above, we define $\beta_{i j, 6}$ as

$$
\beta_{i j, 6}= \begin{cases}0, & v_{i} \geq v_{i}^{0} \\ 1, & v_{i}<v_{i}^{0}\end{cases}
$$

Then, the gravitation model is added to Eq. (1), and the improved model is given as follows:

$$
m_{i} \frac{\mathrm{d} \boldsymbol{v}_{i}}{\mathrm{~d} t}=\boldsymbol{f}_{\text {will }}+\boldsymbol{f}_{i, \text { ped }}+\boldsymbol{f}_{i, \text { wal }}+\boldsymbol{f}_{i, \text { gra }} .
$$

\section{Simulations and discussion}

Simulations are implemented to investigate the impact of following behavior on the process of lane formation, conflict, number of lanes formed, and traffic efficiency. The parameters in the simulations are shown in Table. 1.

\subsection{Impact of following behavior on the pro- cess of lane formation}

To analyze the effect of following behavior on lane formation, lane formation comparisons before and after the introduction of the gravitation model are analyzed from the simulation snapshots. In the simulation, pedestrians are generated at the left and right ends of the passage at the arrival rate of 0.5 persons $/(\mathrm{m} \cdot \mathrm{s})$. The simulation time is $2400 \mathrm{~s}$, and the snapshots are taken during the last $1400 \mathrm{~s}$. In the snapshots, the black circle stands for the pedestrian from the left end of the passage and the white circle for the pedestrian from the right end. The
Table 1 Parameters in simulation

\begin{tabular}{clc}
\hline Symbol & \multicolumn{1}{c}{ Description } & Value \\
\hline$v_{i}^{0}$ & Desire velocity & $1.36 \mathrm{~m} / \mathrm{s}$ \\
$m_{i}$ & Pedestrian mass & $65 \mathrm{~kg}$ \\
$\tau_{i}$ & Characteristic time & $0.5 \mathrm{~s}$ \\
$r_{i}$ & Pedestrian radius & $0.25 \mathrm{~m}$ \\
$A_{i}$ & Avoidance force intensity & $2 \times 10^{3} \mathrm{~N}$ \\
$B_{i}$ & Avoidance coefficient & $0.08 \mathrm{~m}$ \\
$l_{i}$ & Gravitation range & $2 \mathrm{~m}$ \\
$S_{\mathrm{MAX}}$ & Maximum space compression ra- & $20 \%$ \\
& tio of the pedestrian & $1 \mathrm{~m}$ \\
$C_{i}$ & Specified constant & 0.2 \\
$\phi$ & Revise parameter of gravitation & $2.4 \times 10^{4} \mathrm{~kg} / \mathrm{s}^{2}$ \\
$k$ & Body force coefficient & $1 \mathrm{~kg} /(\mathrm{m} \cdot \mathrm{s})$ \\
$\kappa$ & Friction force coefficient & $5 \mathrm{~ms}$ \\
$\Delta t$ & Time step & $0.5 \mathrm{persons} /(\mathrm{m} \cdot \mathrm{s})$ \\
$\lambda$ & Pedestrian arrival rate & $40 \mathrm{~m}$ \\
$L$ & Length of channel & $8 \mathrm{~m}$ \\
$W$ & Width of channel & \\
\hline
\end{tabular}

short line at the front of the circle represents the movement direction of the pedestrian, and the red short line at the front of the circle represents the gravitation direction on the pedestrian.

Fig. 3 shows the lane formation simulation considering the following behavior. As shown, the effect of gravitation is distinct during the weaving process of counter-pedestrian flow. When there is a potential conflict before a pedestrian, the pedestrian immediately slows down to avoid a collision, and the red short line appears; i.e., the gravitation demonstrates its effect. Under the action of gravitation, a pedestrian tends to move to the position of the pedestrian ahead with the same moving direction and follow the routine they left; thus, the collision is avoided. Fig. 4 compares the simulation without and with considering the following behavior. As shown in Fig. 4a, the lanes of pedestrian flow simulated are often broken up by the counter-pedestrian flow if the

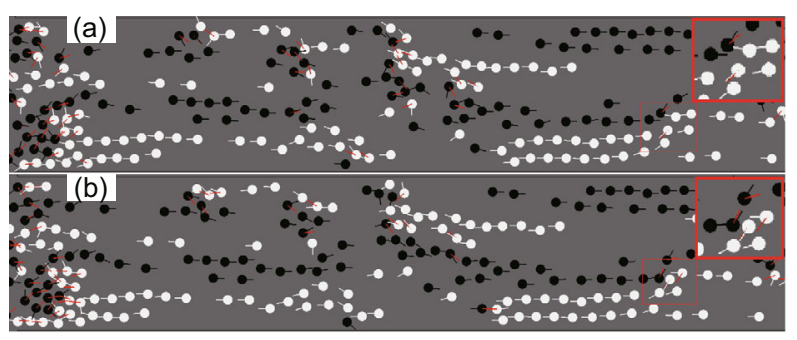

Fig. 3 Snapshots of the lane formation simulation at the time instances $1541 \mathrm{~s}$ (a) and $1543 \mathrm{~s}$ (b) with the following behavior considered. The red regions are magnified in the upper right of each figure (References to color refer to the online version of this figure) 


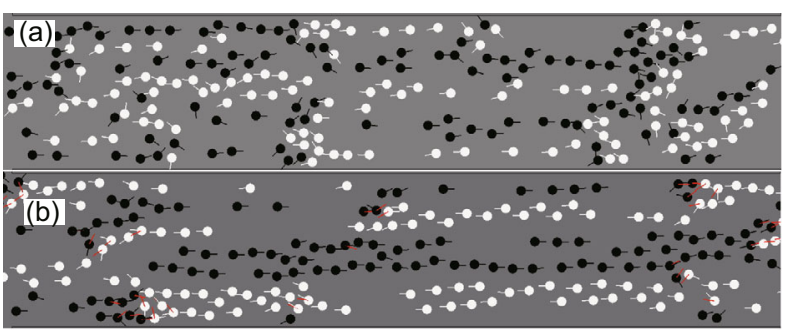

Fig. 4 Comparison of the snapshots of lane formation simulations without (a) and with (b) considering the following behavior (References to color refer to the online version of this figure)

following behavior is not considered, and a traffic jam is locally emergent. If the following behavior is considered, the lane formation is more apparent, the congestion points are fewer, and the weaving of counter-pedestrian flow is more fluent (Fig. 4b).

\subsection{Impact of following behavior on the con- flict between pedestrians}

To further analyze the effect of the following behavior on lane formation, the number of conflicts is counted during the simulation run. In some studies, the conflict is defined as a physical contact between opposite pedestrians. Although in SFM, pedestrians usually do not contact physically because of the existence of the repulsive force, their movement behaviors are affected. The conflict in the study is defined as the case that the distance between two opposite pedestrians which will conflict in non-congested pedestrian flow is less than $5 \mathrm{~cm}$. The relative position where pedestrians conflict is defined as the level of conflict (Fig. 5). The smaller the relative position parameter $l_{i j}$, the more intense the conflict.

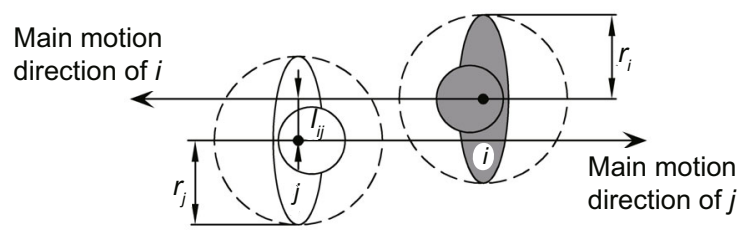

Fig. 5 Illustration of the relative position parameter $l_{i j}$ reflecting the degree of conflict

In the simulation, to study the conflict levels under different degrees of congestion, several arrival rates are set: $0.250,0.375$, and 0.500 persons $/(\mathrm{m} \cdot \mathrm{s})$. The simulations of SFM and SFM with gravitation considered are each run for $140 \mathrm{~s}$ and 200 times, and the average number of conflicts is calculated (Fig. 6). In Fig. 6a, the arrival rate of the free flow is 0.25 persons $/(\mathrm{m} \cdot \mathrm{s})$, and pedestrians walk at their expected speed; therefore, there is almost no following behavior and there is no obvious difference between the number of conflicts with and without considering the following behavior. When the arrival rate increases to 0.375 and 0.500 persons $/(\mathrm{m} \cdot \mathrm{s})$, although the number of conflicts is increasing, SFM with following behavior considered plays clearly a role in reducing the number of conflicts in comparison to SFM without following behavior (Figs. $6 \mathrm{~b}$ and $6 c)$. When $\lambda=0.5$ persons $/(\mathrm{m} \cdot \mathrm{s})$, SFM considering following behavior reduces the number of conflicts in total by $19 \%$ in comparison to SFM. In particular, the intense conflicts are reduced by $29.2 \%$ in the case of $l_{i j}<0.1 \mathrm{~m}$ (Fig. 6c).
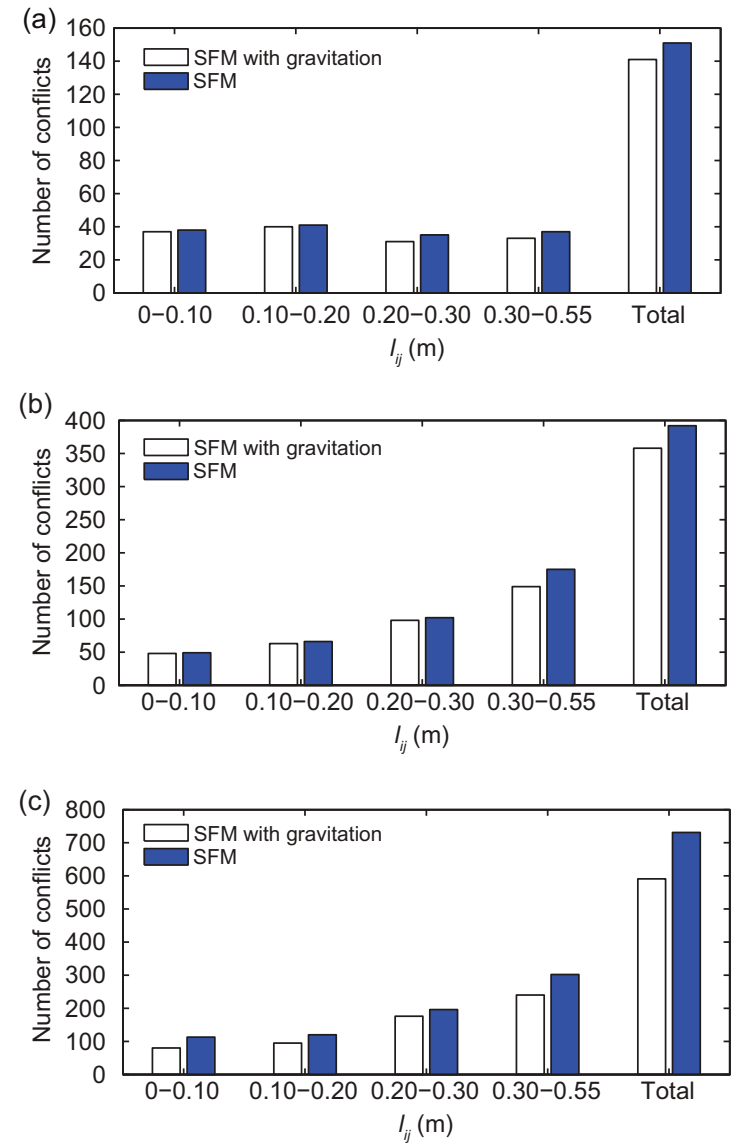

Fig. 6 The relationship between the number of conflicts and the relative position parameter $l_{i j}$ obtained by SFM and SFM with gravitation simulations when the pedestrian arrival rate $\lambda=0.250$ persons $/(\mathrm{m} \cdot \mathrm{s})(\mathrm{a}), \lambda=0.375$ persons $/(\mathrm{m} \cdot \mathrm{s})(\mathrm{b})$, and $\lambda=0.500$ persons $/(\mathrm{m} \cdot \mathrm{s})(\mathrm{c})$ 


\subsection{Impact of following behavior on the num- ber of lanes formed}

The results observed in Ma et al. (2010) indicate that the number of lanes is relatively constant, i.e., about $4-5$ for the whole observation time. In that study, the scenario of lane formation in pedestrian flow is as follows: the region is $8 \mathrm{~m} \times 8 \mathrm{~m}$ and the bidirectional pedestrian density is in the range of $0.83-1.28$ persons $/ \mathrm{m}^{2}$. In this section, the simulation scenario is modeled with similar parameters of the observed pedestrian flow. Five hundred and eighty snapshots are captured and the distribution of the number of lanes formed is obtained.

Fig. 7 is a comparison of the snapshots of lane formation without and with the consideration of following behavior. As shown, the results of simulation without considering following behavior are apparently different from those of the observed scenario, and the numbers of lanes counted from the simulation are between 5 and 8 . The results of simulation considering following behavior are closer to those of the observed scenario, with the number of lanes about 4 or 5 . Fig. 8 is a comparison of the distribution of the numbers of lanes formed without and with the consideration of following behavior. The results show that when considering following behavior, during most of the time the number of lanes keeps at 4 or 5 ( $66 \%$ of all the samples). However, when not considering the following behavior, the range of the number of lanes is even larger (5 lanes account for $31 \%, 6$ lanes account for $24 \%, 7$ lanes account for $24 \%$, and 8 lanes account for $17 \%$ of all the samples). Accordingly, we can find that following behavior can make the pedestrian flow more steady.

\subsection{Impact of following behavior on traffic ef- ficiency of the channel}

To study the effect of following behavior on pedestrian traffic efficiency, the relationship between pedestrian speed and density is drawn for the SFM models and compared with that from empirical data (Older, 1968; Weidmann, 1993) and the field data from our group (Fig. 1). In the simulation, the scenario size is $8 \mathrm{~m}$ (width) $\times 40 \mathrm{~m}$ (length), and pedestrians enter the passage at the same arrival rate from both ends. Different revise parameters of following behavior $\phi$ are input in the model, and the results are shown in Fig. 9. According to Fig. 9, when the fol-
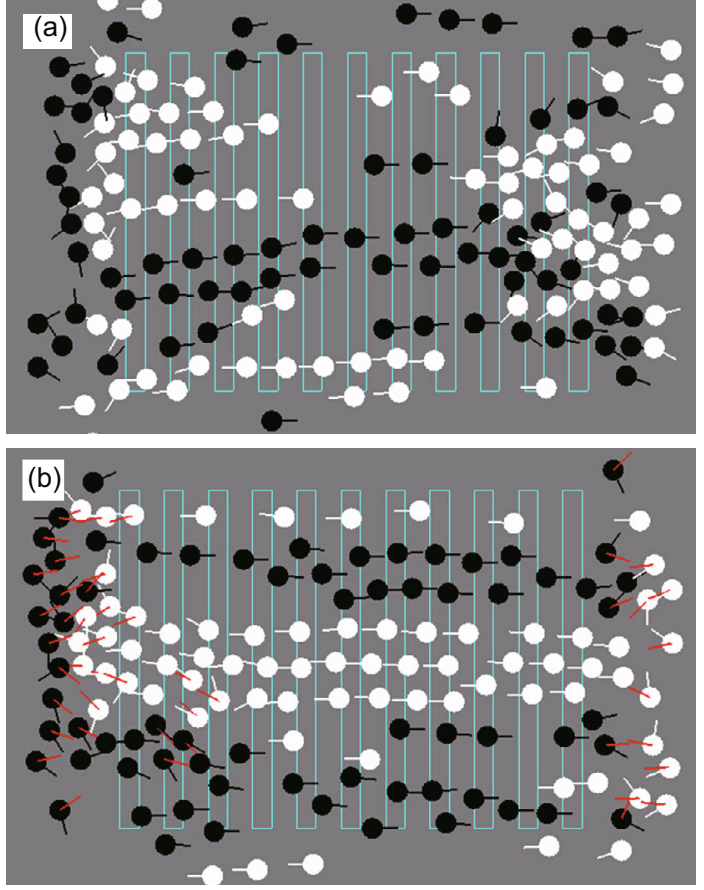

Fig. 7 Snapshots of lane formation simulation in pedestrian crossing counter flow based on the model without (a) and with (b) considering the following behavior
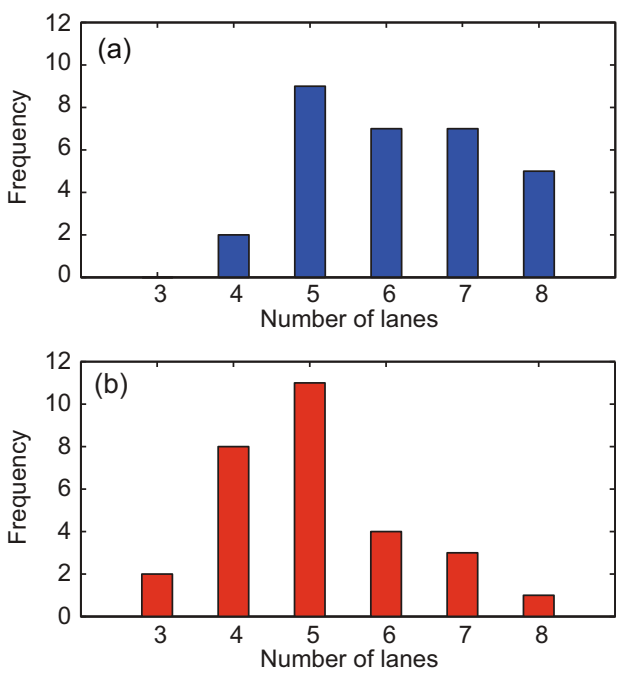

Fig. 8 Distributions of the numbers of lanes formed without (a) and with (b) considering the following behavior

lowing behavior is not modeled, the traffic efficiency is less than that from empirical data. When $\phi=0.2$, the traffic efficiency approaches that from empirical data. The traffic efficiency increases with the increase of the revise parameter of following behavior. This indicates that the following behavior is helpful for increasing the traffic efficiency of the passage, 


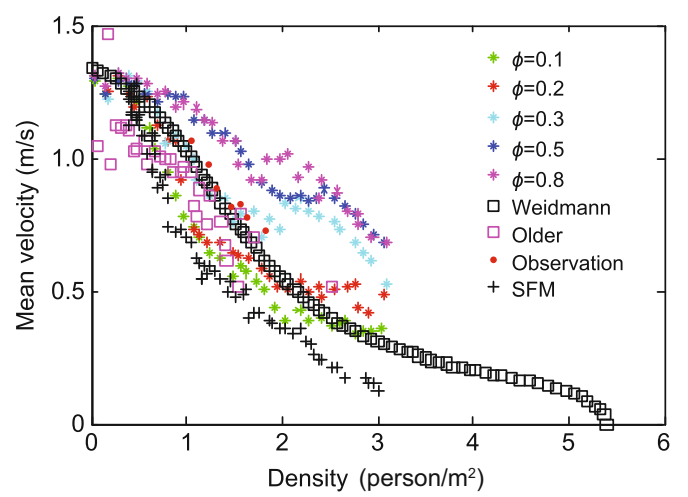

Fig. 9 Fundamental diagram in comparison with experimental data (References to color refer to the online version of this figure)

and the SFM model considering following behavior is more accurate. Because following behavior reflects the will of the pedestrian who follows the pedestrian ahead in the same moving direction, the more intense the will is, the quicker the movement direction of the pedestrian is adjusted, and the quicker the lane is formed. Thus, the traffic efficiency is increased.

\section{Conclusions}

In the paper, a new pedestrian flow model with gravitation is proposed to simulate following behavior and the lane formation phenomenon. The gravitation is of the same order as the willing force and its direction is from the location of the current pedestrian to that of the followed pedestrian. Thus, a new pedestrian model is built based on gravitation. In our simulation, we analyze the differences of the macroeconomic phenomenon and the impact of following behavior on the conflict, the number of lanes formed, and the traffic efficiency with the classical model. It is found that following behavior is helpful in avoiding unnecessary conflicts and in easing the conflicts, and it is conducive to improving the efficiency of the passage. Other self-organizing phenomena like stripe formation and following behavior in the state of panic will be studied in the future.

\section{Acknowledgements}

We would like to thank Dr. Lei BIAN and Dr. Yongjun MA for the stimulating discussions.

\section{References}

Chen, M.J., Bärwolff, G., Schwandt, H., 2009. A derived grid-based model for simulation of pedestrian flow. $J$. Zhejiang Univ.-Sci. A, 10(2):209-220. https://doi.org/10.1631/jzus.A0820049
Fujiyama, T., Tyler, N., 2009. Bidirectional collisionavoidance behaviour of pedestrians on stairs. Environ. Plan. B, 36(1):128-148. https://doi.org/10.1068/b33123

Guo, R.Y., 2014. Simulation of spatial and temporal separation of pedestrian counter flow through a bottleneck. Phys. A, 415:428-439. https://doi.org/10.1016/j.physa.2014.08.036

Helbing, D., 1996. A stochastic behavioral model and a 'microscopic' foundation of evolutionary game theory. Theory Dec., 40(2):149-179. https://doi.org/10.1007/BF00133171

Helbing, D., 2001. Traffic and related self-driven manyparticle systems. Rev. Mod. Phys., 73(4):1067. https://doi.org/10.1103/RevModPhys.73.1067

Helbing, D., Molnár, P., 1995. Social force model for pedestrian dynamics. Phys. Rev. E, 51(5):4282. https://doi.org/10.1103/PhysRevE.51.4282

Helbing, D., Farkas, I., Vicsek, T., 2000. Simulating dynamical features of escape panic. Nature, 407(6803):487-490. https://doi.org/10.1038/35035023

Helbing, D., Farkas, I.J., Molnár, P., et al., 2002. Simulation of pedestrian crowds in normal and evacuation situations. Proc. 1st Int. Conf. on Pedestrian and Evacuation Dynamics, p.21-58.

Helbing, D., Buzna, L., Johansson, A., et al., 2005. Selforganized pedestrian crowd dynamics: experiments, simulations, and design solutions. Transp. Sci., 39(1):1-24. https://doi.org/10.1287/trsc.1040.0108

Heliövaara, S., Korhonen, T., Hostikka, S., et al., 2012. Counterflow model for agent-based simulation of crowd dynamics. Build. Environ., 48:89-100. https://doi.org/10.1016/j.buildenv.2011.08.020

Iryo-Asano, M., Alhajyaseen, W.K.M., Nakamura, H., 2015. Analysis and modeling of pedestrian crossing behavior during the pedestrian flashing green interval. IEEE Trans. Intell. Transp. Syst., 16(2):958-969. https://doi.org/10.1109/TITS.2014.2346154

Jia, H.F., Li, Y.X., Yang, L.L., et al., 2016. Modeling the separating pedestrian flow in T-shaped passage based on guide sign. Discr. Dynam. Nat. Soc., 2016:5625286. https://doi.org/10.1155/2016/5625286

Kuang, H., Li, X.L., Wei, Y.F., et al., 2010. Effect of following strength on pedestrian counter flow. Chin. Phys. B, 19(7):070517. https://doi.org/10.1088/1674-1056/19/7/070517

Lakoba, T.I., Kaup, D.J., Finkelstein, N.M., 2005. Modifications of the Helbing-Molnár-Farkas-Vicsek social force model for pedestrian evolution. Simulation, 81(5):339352. https://doi.org/10.1177/0037549705052772

Lam, W.H.K., Lee, J.Y.S., Cheung, C.Y., 2002. A study of the bi-directional pedestrian flow characteristics at Hong Kong signalized crosswalk facilities. Transportation, 29(2):169-192. https://doi.org/10.1023/A:1014226416702

Li, J., Yang, L., Zhao, D., 2005. Simulation of bi-direction pedestrian movement in corridor. Phys. A, 354:619628. https://doi.org/10.1016/j.physa.2005.03.007

Li, J., Wang, J., Dong, Y., et al., 2015. Streamline simulation and analysis of pedestrian weaving flow in large passenger terminal. Math. Probl. Eng., 2015:645989. https://doi.org/10.1155/2015/645989 
Li, Y.X., Jia, H.F., Zhou, Y.N., et al., 2017. Simulation research on pedestrian counter flow subconscious behavior. Int. J. Mod. Phys. C, 28(2):1750025. https://doi.org/10.1142/S0129183117500255

Liao, M.J., Liu, G., 2015. Modeling passenger behavior in nonpayment areas at rail transit stations. Transp. Res. Rec. J. Transp. Res. Board, 2534:101-108. https://doi.org/10.3141/2534-13

Löhner, R., 2010. On the modeling of pedestrian motion. Appl. Math. Model., 34(2):366-382. https://doi.org/10.1016/j.apm.2009.04.017

Ma, J., Song, W.G., Zhang, J., et al., 2010. $k$-nearestneighbor interaction induced self-organized pedestrian counter flow. Phys. A, 389(10):2101-2117. https://doi.org/10.1016/j.physa.2010.01.014

Older, S.J., 1968. Movement of pedestrians on footways in shopping streets. Traff. Eng. Contr., 10(4):160-163.

Pelechano, N., Allbeck, J.M., Badler, N.I., 2007. Controlling individual agents in high-density crowd simulation. Proc. ACM SIGGRAPH/Eurographics Symp. on Computer Animation, p.99-108. https://doi.org/10.2312/SCA/SCA07/099-108

Saloma, C., Perez, G.J., Tapang, G., et al., 2003. Selforganized queuing and scale-free behavior in real escape panic. PNAS, 100(21):11947-11952. https://doi.org/10.1073/pnas.2031912100

Seyfried, A., Steffen, B., Klingsch, W., et al., 2005. The fundamental diagram of pedestrian movement revisited. J. Stat. Mech. Theory Exp., 2005(10):P10002. https://doi.org/10.1088/1742-5468/2005/10/P10002

Smith, A., James, C., Jones, R., et al., 2009. Modelling contra-flow in crowd dynamics DEM simulation. Safety Sci., 47(3):395-404. https://doi.org/10.1016/j.ssci.2008.05.006

Tajima, Y., Takimoto, K., Nagatani, T., 2002. Pattern formation and jamming transition in pedestrian counter flow. Phys. A, 313(3):709-723.

https://doi.org/10.1016/S0378-4371(02)00965-2

Tang, T.Q., Shao, Y.X., Chen, L., 2017. Modeling pedestrian movement at the hall of high-speed railway station during the check-in process. Phys. A, 467:157-166. https://doi.org/10.1016/j.physa.2016.10.008

Wang, Z., Ma, J., Zhao, H., et al., 2012. Effect of prediction on the self-organization of pedestrian counter flow. $J$. Phys. A, 45(30):305004. https://doi.org/10.1088/1751-8113/45/30/305004

Weidmann, U., 1993. Transporttechnik der Fussgänger: Transporttechnische Eigenschaften des Fussgängerverkehrs (Literaturauswertung). ETH Zürich (in German). https://doi.org/10.3929/ethz-a-000687810

Weng, W.G., Chen, T., Yuan, H.Y., et al., 2006. Cellular automaton simulation of pedestrian counter flow with different walk velocities. Phys. Rev. E, 74(3):036102. https://doi.org/10.1103/PhysRevE.74.036102

Werner, T., Helbing, D., 2003. The social force pedestrian model applied to real life scenarios. Proc. 2nd Int. Conf. on Pedestrian and Evacuation Dynamics, p.1726.

Yang, L., Li, J., Liu, S., 2008. Simulation of pedestrian counter-flow with right-moving preference. Phys. A, 387(13):3281-3289. https://doi.org/10.1016/j.physa.2008.01.107

Yue, H., Guan, H., Zhang, J., et al., 2010. Study on bidirection pedestrian flow using cellular automata simulation. Phys. A, 389(3):527-539. https://doi.org/10.1016/j.physa.2009.09.035

Zhang, J., Wang, H., Li, P., 2004. Cellular automata modeling of pedestrian's crossing dynamics. J. Zhejiang Univ.-Sci., 5(7):835-840. https://doi.org/10.1631/jzus.2004.0835 